Questions vives

\section{Questions Vives}

Recherches en éducation

Vol.9 $n^{\circ} 19$ | 2013

Éducation routière, changement de comportement et formation à la conduite : constat, enjeux et transformations

\title{
Quelles innovations pédagogiques dans le cadre de la réforme du permis de conduire?
}

Comment appliquer la matrice GDE ? Quelles adaptations aux facteurs de risque d'accidents des jeunes conducteurs novices?

Which pedagogical innovations in the improvement of the licensing process?

How to apply the GDE matrix? How is it adapted to the accident risk factors of young novice drivers?

\section{Assailly Jean-Pascal}

\section{(2) OpenEdition}

Journals

Édition électronique

URL : http://journals.openedition.org/questionsvives/1268

DOI : $10.4000 / q u e s t i o n s v i v e s .1268$

ISBN : 978-2-8218-1394-6

ISSN : $1775-433 X$

Éditeur

Université Aix-Marseille (AMU)

Édition imprimée

Date de publication : 15 juillet 2013

Pagination : 37-49

ISBN : 978-2-912643-43-8

ISSN : 1635-4079

Référence électronique

Assailly Jean-Pascal, « Quelles innovations pédagogiques dans le cadre de la réforme du permis de conduire? », Questions Vives [En ligne], Vol. 9 n $^{\circ} 19$ | 2013, mis en ligne le 15 juillet 2013, consulté le 03 mai 2019. URL : http://journals.openedition.org/questionsvives/1268 ; DOI : 10.4000/ questionsvives. 1268

Ce document a été généré automatiquement le 3 mai 2019.

Questions Vives est mis à disposition selon les termes de la licence Creative Commons Attribution Pas d'Utilisation Commerciale - Pas de Modification 4.0 International. 


\section{Quelles innovations pédagogiques dans le cadre de la réforme du permis de conduire?}

Comment appliquer la matrice GDE ? Quelles adaptations aux facteurs de risque d'accidents des jeunes conducteurs novices?

Which pedagogical innovations in the improvement of the licensing process?

How to apply the GDE matrix? How is it adapted to the accident risk factors of young novice drivers?

Assailly Jean-Pascal

\section{Introduction}

1 L'un des défis universels posé au secteur de la formation des conducteurs est le sur-risque accidentel des jeunes conducteurs novices : en 2011 (dernières données disponibles), les $18-24$ ans constituent $9 \%$ de la population, $21 \%$ des tués (813 tués sur 3963 ) et $20 \%$ des blessés hospitalisés (6039 sur 29 679) en France en 2011 (ONISR, 2011). Ce sur-risque s'observe dans tous les pays, donc dans des systèmes culturels, politiques, religieux, sociaux et réglementaires d'accès à la conduite très différents (Assailly, 2001, 2007, 2010) et depuis les débuts du recueil de données d'insécurité routière dans notre pays (1955). Cette constance du sur-risque partout et depuis toujours, quel que soit le système de formation mis en place, pourrait nous amener à conclure que la formation des conducteurs est impuissante à contrer ce phénomène.

2 Un débat persiste depuis les débuts de la recherche en sécurité routière sur les importances respectives des deux facteurs de ce sur-risque, l'âge et l'inexpérience; en effet, si l'âge était en seul en cause (les caractéristiques biologiques, psychologiques et sociales de l'adolescence et de la post-adolescence, le décalage dans l'agenda neurobiologique entre la maturation du système de récompense des aires limbiques et 
celle du système de contrôle cognitif des décisions des aires préfrontales, cf. Assailly, 2010), la formation des conducteurs serait a priori peu opérante et efficace; si l'inexpérience des jeunes conducteurs novices joue un rôle, la formation peut espérer avoir plus d'influence. Or, il semble bien que l'inexpérience joue également: les conducteurs novices ont un risque plus important d'accident dans les 6 premiers mois quel que soit leur âge (Mayhew et al., 2003), même si les jeunes conducteurs ont évidemment un risque plus important que les adultes novices.

Diverses stratégies ont été appliquées pour essayer de réduire ce sur-risque accidentel des jeunes conducteurs novices: en Amérique du Nord et en Australie, la stratégie dominante est de réduire l'exposition au risque lors de ces années d'inexpérience de la conduite par des systèmes dits "d'accès graduel à la conduite » ou de "permis progressif ». Dans ces pays où les transports en commun et la mobilité en deux-roues sont peu développés, les jeunes accèdent plus précocement à la conduite (entre 14 et 18 ans) car ils sont plus captifs et dépendants de la voiture. L'idée est de leur interdire au début un certain nombre de droits (conduite de nuit, avec des passagers, avec alcool, sur autoroute, etc.), puis au bout d'un certain nombre d'années (permis probatoire d'apprenti), si le jeune ne commet pas d'infractions et n'est pas impliqué dans des accidents, il obtient le permis de plein droit (en Nouvelle Zélande par exemple, les jeunes sortent de ce " parcours du combattant » à 25 ans...). Le jeune parcourt ainsi ses premiers milliers kilomètres dans des situations de moindre risque puisque les facteurs de risque de l'accident mortel ont été ciblés (nuit, alcool, cannabis, fatigue, pression des passagers, vitesse, cf. Assailly, 2001).

Dans tous les pays où ce système d'accès graduel à la conduite a été évalué, on a observé effectivement des diminutions significatives des taux d'accidents des jeunes conducteurs novices (voir la revue de la littérature de Russell et al., 2011) ; toutefois, l'accès graduel à la conduite n'abolit pas le sur-risque des jeunes conducteurs, et comme toutes les mesures de sécurité routière, même les meilleures, il finit par atteindre son asymptote d'efficacité et d'acceptabilité sociale; on ne peut "enfermer» les jeunes pour les protéger, d'autres enjeux que l'insécurité routière existent pour cette cible (l'insertion, l'autonomie, la socialisation, etc.). L'accès graduel va par ailleurs être plus difficilement acceptable socialement dans les pays européens où l'accès au permis est plus tardif, alors que les jeunes sont majeurs.

5 En fait, l'une des raisons au développement de l'accès graduel à la conduite provient précisément des évaluations des formations des jeunes conducteurs réalisées dans les universités américaines dans les années 80, et notamment de celle du programme du De Kalb County en Géorgie par la NHTSA (National Highway Traffic Safety Administration) : 16000 étudiants avaient été assignés de manière aléatoire à un programme d'entraînement de 20 heures (en classe, sur simulateur, sur route) ou à un groupe contrôle ; si le programme avait amélioré les compétences de conduite, il ne se traduisait pas par un bénéfice préventif en ce qui concerne les accidents; les sujets entraînés avaient même un taux supérieur d'accidents (Lund et al., 1986) car le programme produisait des accès au permis plus précoces et des expositions au risque plus importantes!

6 Depuis, la majorité des collègues anglo-saxons ont conclu que «l'éducation routière ne marche pas», que «la formation est contre-productive», ce qui a progressivement conforté la seule option de l'accès graduel et l'idée qu'il fallait protéger les jeunes malgré eux! La question qui subsiste est celle-ci : quel type de formation des conducteurs avait 
été évalué dans ces années 80 pour conclure que la formation est inefficace ? S'il s'agissait de formations uniquement centrées sur le pilotage alors que les facteurs de risque sont plutôt situés dans les styles de vie, il n'est pas surprenant d'avoir observé peu d'effets préventifs! Comme si l'on voulait évaluer l'influence de cours de natation sur la performance en hockey sur glace! Ce problème se pose aussi à propos de l'évaluation de l'efficacité préventive des formations dispensées actuellement dans les auto-écoles de notre pays!

7 La question est donc de savoir quelles compétences la formation est-elle censé améliorer, et quel modèle théorique du comportement de conduite automobile sous-jacent oriente les actions et les recherches dans ce domaine. Ainsi, le concept «d'éducation du conducteur » se veut plus large que celui de "formation du conducteur ", il l'incorpore et y ajoute d'autres objectifs, contenus et méthodes.

8 Aux débuts des années 90, un groupe de chercheurs européens (Hatakka et al., 2002) a proposé un nouveau modèle théorique du comportement de conduite, dit "modèle hiérarchique » en 5 niveaux, et une matrice d'objectifs pédagogique correspondant à ce modèle théorique, dite "matrice GDE» (Goals of Driver Education, ou objectifs de l'éducation du conducteur, voir la matrice ci-après). Les objectifs de cette matrice supposent de développer trois types de compétences (des savoirs et des savoir-faire, la compréhension des facteurs de risque qui dégradent le comportement, des compétences d'autoévaluation). On passe ainsi du "contrôle du véhicule» au "contrôle de la personne ».

Figure 1: La matrice GDE

\begin{tabular}{|l|c|c|c|}
\hline & $\begin{array}{c}\text { Savoirs et } \\
\text { savoir-faire }\end{array}$ & Facteurs de risque & Autoévaluation \\
\hline $\begin{array}{l}\text { Pressions sociétales, } \\
\text { économiques }\end{array}$ & V.1 & V.2 & V.3 \\
\hline $\begin{array}{l}\text { Objectifs existentiels } \\
\text { et contrôle de soi }\end{array}$ & IV.1 & IV.2 & IV.3 \\
\hline $\begin{array}{l}\text { Objectifs de } \\
\text { la conduite }\end{array}$ & III.1 & III.2 & III.3 \\
\hline $\begin{array}{l}\text { Compréhension des } \\
\text { scenarii routiers }\end{array}$ & II.1 & II.2 & II.3 \\
\hline $\begin{array}{l}\text { Maîtrise des } \\
\text { manœuvres }\end{array}$ & I.1 & I.2 & I.3 \\
\hline
\end{tabular}

La matrice n'est pas un programme de formation au sens traditionnel du terme, elle ne fixe pas de contenus mais décrit des processus (les pressions cybernétiques des niveaux supérieurs de la conduite qui produisent des contraintes d'organisation sur les niveaux inférieurs) et laisse vides, ouvertes les 15 cases des objectifs pédagogiques. Illustrons ce modèle par un exemple : si le sujet présente une certaine vulnérabilité au 5ème niveau, 
celui des pressions sociétales et économiques (par exemple de type sensibilité aux publicités associant grande vitesse et érotisme ou popularité), cette vulnérabilité peut créer un problème au 4ème niveau, celui du contrôle de soi (dépendance croissante vis-àvis de la vitesse pour libérer sa dopamine ou renforcer l'estime de soi), ce problème du 4ème niveau produira des contraintes au 3ème niveau, celui du rapport à la conduite automobile (posséder un véhicule très puissant et rapide, rechercher des situations routières permettant la grande vitesse), ces contraintes du 3ème niveau imposeront des pressions organisationnelles sur les compétences du 2ème niveau, celui de la compréhension des scénarios d'accident et des situations de conduite (devoir analyser très rapidement les indices de danger), ces pressions du second niveau dégraderont enfin les performances du premier niveau, celui de la réussite des manœuvres (ce qui augmentera la probabilité de l'accident lors de l'échec d'une réalisation).

Cette vision cybernétique nous permet de comprendre par exemple pourquoi les femmes réussissent moins l'examen du permis de conduire en première passation mais ont moins d'accidents que les hommes (bien que moins performantes sur les deux niveaux inférieurs de la matrice, elles sont moins déficientes sur les trois niveaux supérieurs), pourquoi des conducteurs peu compétents sur le pilotage peuvent avoir un meilleur bilan accidentel que des conducteurs plus compétents, ou enfin pourquoi certaines formations trop axées sur le premier niveau peuvent se révéler contre-performantes (effet de rétroaction circulaire négatif de l'entraînement des niveaux inférieurs sur les niveaux supérieurs, produisant de la surconfiance).

La matrice suppose très probablement des partenariats entre divers secteurs car il est peu probable de trouver un formateur qui serait compétent pour «balayer » les 15 cases de la matrice à lui seul!

Elle suppose enfin d'impulser pour chaque objectif pédagogique introduit dans les cases une interaction cybernétique décrite par une «figure des 4 coins » (reproduite dans la figure 2 ci-après) : face à un nouvel objectif introduit dans la formation, il faut un formateur compétent, ce dernier doit employer une méthode pédagogique adaptée, et la formation doit être évaluée, testée à la fin du processus.

Figure 2 : La « figure des 4 coins »

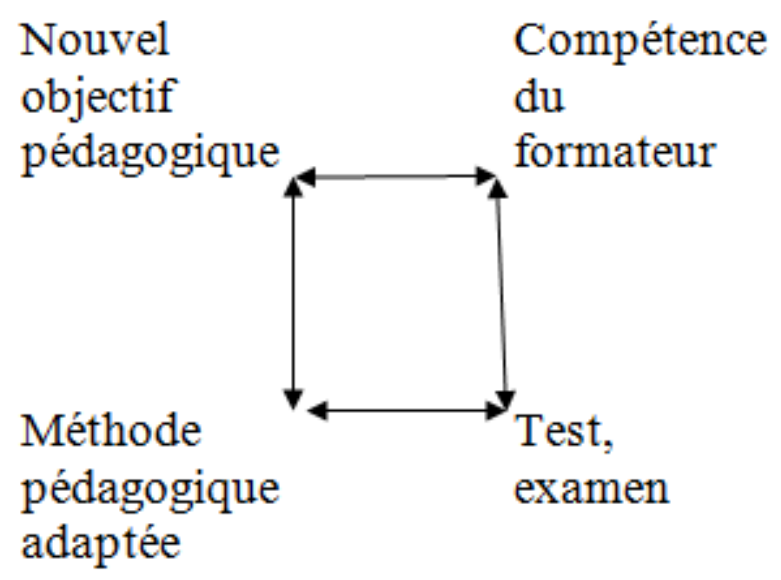

Depuis l'élaboration des modèles hiérarchiques du comportement de conduite et de la matrice GDE, divers programmes de formation ont été impulsés et évalués afin d'améliorer la sécurité des jeunes conducteurs novices; nous nous proposons dans cet 
article de présenter l'état de la connaissance sur ces questions, en distinguant les formations avant et après le permis.

\section{Les formations avant le permis}

14 Elles concernent le plus souvent les deux niveaux inférieurs de la matrice, concernant la performance (la maîtrise des manœuvres et la compréhension des situations accidentogènes). Elles sont de trois types :

\subsection{La formation par des formateurs professionnels (enseignants de la conduite)}

Cette formation est prépondérante dans notre pays et peut être marginale dans d'autres ; on estime généralement qu'elle facilite l'obtention du permis, via le succès à l'examen pratique (Nyberg et al., 2007). Les compétences du premier niveau (la maîtrise des manœuvres) sont donc améliorées par cette formation et le gain sécuritaire est obtenu à condition qu'elles ne produisent pas de surconfiance et d'optimisme comparatif (Assailly, 2010). Ainsi, une évaluation au Danemark de l'introduction de l'obligation d'un cours de conduite avec des formateurs professionnels, qui comprenait des formations en classe, de l'entrainement aux manœuvres, de la conduite défensive et de l'entraînement à la perception des dangers (Carstensen, 2002), a montré un effet sur une diminution des taux d'accidents. En Norvège, la formation par des formateurs professionnels a été associée à des attitudes plus sécuritaires, des infractions moins fréquentes et une diminution de la surconfiance (Tronsmoen, 2010).

De telles évaluations comparant formations professionnelles et non professionnelles n'ont jamais été menées dans notre pays, et l'hétérogénéité de la qualité d'un établissement à l'autre serait un facteur à prendre en compte.

\subsection{Les formations à la conduite en milieu scolaire et universitaire}

17 Comme indiqué plus haut, les évaluations des formations en milieu scolaire et universitaire ont été plutôt négatives (Lund et al., op.cit.) du fait des effets pervers observés.

\subsection{Les formations sur simulateur}

18 Elles ont été particulièrement développées aux Pays-Bas et sont fondées sur l'idée que le simulateur peut fournir une alternative à l'entraînement pratique sur route en permettant à l'élève de se confronter à des situations d'urgence et à une grande variété de situations routières sans se mettre en danger (de Winter et al., 2007). Peu de travaux ont évalué l'efficacité de cette stratégie : elle peut améliorer les compétences pratiques, mais il n'y a pas toujours généralisation de l'apprentissage à d'autres compétences dans d'autres contextes, elle peut diminuer le risque accidentel mais uniquement avec les appareils haut de gamme (véhicule complet, champ visuel à $180^{\circ}$ ) selon Allen et al. (2007). On peut penser en effet que plus le stimulus virtuel se rapproche du réel, meilleure sera l'efficacité, mais ce champ souffre encore de carences méthodologiques importantes (biais de sélection des échantillons et absence de groupes témoins). 


\subsection{L'accès graduel à la conduite} globalement, les restrictions imposées par l'accès graduel se traduisent par une diminution de l'implication accidentelle des jeunes conducteurs novices comme nous l'avons évoqué plus haut; toutefois, la motivation des jeunes apprentis sous-jacente au fait de prendre des cours de conduite a aussi un effet: ainsi, les jeunes conducteurs novices Québécois qui prenaient des leçons afin d'améliorer leurs compétences avaient des taux plus bas d'accidents et d'infractions que ceux dont la motivation étaient d'accélérer l'accès au permis ou d'obtenir une réduction de la prime d'assurance (Hirsch et al., 2006). Les motivations et les attitudes pourraient donc jouer comme des facteurs de confusion lors des évaluations des programmes de formation.

\subsection{La conduite accompagnée}

nouveau, il ne s'agit pas d'une formation mais d'un renforcement de l'expérience de conduite par l'obligation (théorique) des $3000 \mathrm{~km}$ avec le parent accompagnateur, où certes des compétences et des attitudes peuvent être transmises par le "parent formateur ». Les principales observations sont désormais bien connues: difficulté de la diffusion de la mesure pour des jeunes dont la situation socio-familiale ne le permet pas ; facilitation de l'obtention du permis ( $70 \%$ de réussite en première passation contre $50 \%$ en formation traditionnelle); bénéfice préventif sur l'implication accidentelle qui peut être mitigé par divers facteurs : hétérogénéité de la qualité et du respect du cahier des charges d'un établissement à l'autre, absence de formation et de sélection des accompagnateurs, etc.

\section{Les formations après le permis}

Elles peuvent théoriquement concerner tous les niveaux de la matrice et sont de deux types:

\subsection{Les formations pratiques}

Elles visent à améliorer les compétences techniques (freinage, dérapage, etc.) du premier niveau de la matrice. Certaines évaluations ont montré des effets positifs mais spécifiques et à court terme: par exemple, un programme d'une semaine en Nouvelle Zélande a amélioré l'adaptation du choix de la vitesse mais non pas la perception des dangers ou les attitudes relatives au risque (Isler et al., 2011). De même, plusieurs études australiennes ont observé des effets positifs de programmes de deux jours sur le freinage et le contrôle du véhicule, mais là encore sans généralisation de l'apprentissage à divers contextes : par exemple, les jeunes conducteurs novices ayant suivi la formation mettaient plus de temps à freiner sur un véhicule équipé d'ABS que des conducteurs non formés (Petersen et al., 2006).

Parmi les diverses compétences techniques, le dérapage a fait l'objet d'une attention plus particulière, depuis l'abandon de la formation au dérapage dans les pays scandinaves: alors qu'il pouvait sembler logique d'imposer l'obligation de ce type de formation dans 
ces pays où les routes sont souvent glacées et enneigées, cette obligation a été progressivement abandonnée du fait de ses effets pervers (les conducteurs formés à conduire sur glace présentaient des taux plus élevés d'accidents... sur glace que les conducteurs non formés, du fait du phénomène de surconfiance); ainsi, il a été observé que les jeunes se centraient plus sur la réussite de la manœuvre que sur l'évitement du danger (Katila et al., 1996) et que cet entraînement accroissait l'écart entre l'autoévaluation de ses compétences en matière de dérapage et ses compétences réelles, ce qui produit la surconfiance (Gregersen, 1996).

Les experts scandinaves ont donc proposé l'abandon de cette stratégie ou sa réforme, par exemple en centrant l'entraînement sur l'évitement du danger et en structurant les exercices de manière à ce qu'ils ne puissent être réussis à des vitesses dangereuses (certains exercices en Suède consistent par exemple à mettre le jeune en échec afin qu'il réduise sa vitesse de lui-même, améliorant ainsi l'autoévaluation); toutefois, même en focalisant sur l'évitement du danger, des évaluations ultérieures (Katila et al., 2004) ont montré un décalage entre les conceptions des formateurs (l'évitement) et celles des jeunes (attente de maîtrise).

25 Enfin, une évaluation randomisée a été réalisée dans le Montana à propos des effets d'une formation d'une journée à la conduite défensive (manœuvres d'urgence, dérapage, contrôle du véhicule) sur 347 jeunes conducteurs novices (Kelly, 2005); un suivi sur 4 ans ne montre pas d'effets sur les accidents ou les infractions.

\subsection{Les formations cognitives}

26 Elles visent à améliorer les compétences nécessaires au second niveau de la matrice, l'anticipation et la compréhension des situations routières dangereuses et des scénarii d'accidents.

27 - L'entraînement à la perception des dangers : Cette stratégie a été particulièrement développée en Angleterre du fait des nombreux travaux menés dans ce pays sur les relations entre perception des dangers et risque d'accident: en effet, les conducteurs novices ont des moins bonnes compétences dans ces domaines que les conducteurs expérimentés, leurs explorations visuelles sont moins efficaces, ils regardent moins les rétroviseurs extérieurs, et leur champ de vision périphérique est plus réduit (Underwood et al., 2002). Les experts anglais ont donc développé des tests de perception des dangers à partir de vidéos où l'élève doit détecter des indices de danger et prendre des décisions, et montré que les résultats de ces tests étaient prédictifs de l'implication accidentelle lors de la première année de la conduite (Boufous et al., 2011).

28 La question est donc de savoir si l'entraînement à la perception des dangers peut améliorer cette compétence ; deux types différents de formation ont été évalués :

29 - La conduite commentée : Cette formation consiste à fournir des commentaires aux élèves lorsqu'ils conduisent ou lorsqu'ils regardent une vidéo ; les jeunes conducteurs qui ont suivi cette formation ont de meilleurs scores aux tests de perception des dangers, améliorent leurs explorations visuelles périphériques et réduisent plus leurs vitesses lorsqu'ils approchent d'un danger dans les tests sur simulateur (Crundall et al., 2010).

Cette approche améliore le respect des distances de sécurité chez les conducteurs formés, ceci peut être considéré comme une amélioration des comportements dangereux et infractionnistes, du moins de ceux qui sont liés à l'inexpérience et aux 
dysfonctionnements de la perception du danger (et non pas de ceux qui sont liés à l'âge et aux prises de risque délibérées, cf. Assailly, 2010).

31 Une approche similaire a consisté à exposer les jeunes conducteurs à une série de situations dangereuses sur simulateur, puis l'élève formé visionne de nouveau une vidéo de sa performance en la comparant à celle d'un expert (Wang et al., 2010). Cet entraînement améliore l'anticipation des dangers.

32 - Le complètement de tâche : Dans cette approche, les participants doivent regarder des vidéos et compléter les tâches comme prédire les dangers possibles ou indiquer des actions appropriées; les sujets formés ont des performances améliorées, leurs explorations visuelles sont meilleures (ils fixent plus les zones de danger potentiel, que ce soit sur simulateur ou sur route (Fisher et al., 2006).

Ces résultats sont donc encourageants mais, comme pour la conduite commentée, nous manquons de données pour savoir si ces progrès se traduisent réellement en termes d'accidents.

Pour conclure, sur ces formations post-permis, nous pouvons dire que les formations pratiques améliorent les compétences techniques mais que le gain de sécurité n'est pas évident car les jeunes conducteurs novices ne vont pas ensuite utiliser correctement ces savoirs-faire, et que les formations cognitives semblent bien améliorer la prise de conscience des indices de danger (et ceci plus pour des conducteurs novices que pour des conducteurs expérimentés) sans que des données probantes sur l'implication accidentelle soient encore disponibles.

\subsection{Les formations sur les compétences de vie}

35 Plus récemment sont apparues des formations visant le quatrième niveau de la matrice, c'est-à-dire le développement de compétences visant la personne et non plus le conducteur, intitulées "compétences de vie»(life-skills): il s'agit, entre autres, du renforcement de l'estime de soi, de l'assertivité ou affirmation de soi en groupe, des capacités de résistance aux pressions, du contrôle de soi, de l'efficacité perçue de soi, etc. On peut penser aussi aux compétences dîtes «méta-cognitives» telles que l'autoévaluation. Bref, ces formations n'ont plus aucun contenu en rapport direct avec la tâche de conduite d'une voiture.

Deux exemples récents de tels programmes en milieu scolaire, évalués ou en cours d'évaluation:

- le programme You Hold the Key (YHTK) développé dans l'Ohio en direction des conducteurs adolescents de 15/19 ans (qui sont déjà conducteurs de voiture dans ce pays); il dure dix semaines et comporte une grande variété d'activités : des présentations powerpoint, des interventions de policiers et de juges sur l'infraction-alcool, des panels de discussion avec des jeunes adultes, des témoignages de victimes, des vidéos sur diverses thématiques de l'insécurité routière des jeunes.

- le programme ESPACE à Poitiers visant à favoriser une consommation d'alcool socialement responsable, par deux objectifs (retarder l'âge de début de la consommation régulière d'alcool ; retarder l'âge de début des ivresses), en direction de 1200 collégiens. Il est centré sur l'amélioration des compétences de vie, de l'autoévaluation et de l'estime de soi, et il utilise des jeux de rôle et des discussions de groupe. 

au bien-être des jeunes, car elles posent que les états de détresse psychologique sont bien souvent au cœur de la genèse des phénomènes de prise de risques, d'addiction et de transgression (Assailly, 2007). Leur difficulté réside dans le temps, l'investissement et l'engagement qu'elles supposent, ce qui limite pour le moment le nombre de tentatives.

\subsubsection{Les entraînements des « compétences de vie »}

Les évaluations menées aux USA et en Australie ont conclu à des effets positifs de telles formations : augmentation du taux de port de la ceinture, de la capacité d'éviter les situations de conduite sous influence par le programme You Hold the Key (King et al., 2008), diminution des taux d'accidents par un programme d'intervention communautaire en Australie (Senserrick et al., 2009). En France, le programme ESPACE a déjà montré des impacts positifs sur l'estime de soi et les compétences de vie (capacité de résister à la pression du groupe) des 11/13 ans.

\subsubsection{Les programmes d'amélioration de l'autoévaluation (diminution de la surconfiance ou insight)}

Deux méthodes usitées consistent à demander au sujet de se comparer à un conducteur " moyen », ou bien de comparer son autoévaluation à celle d'un instructeur ; on peut ainsi dégager la prévalence de la surconfiance ou de l'optimisme comparatif : de 30 à $40 \%$ des jeunes conducteurs novices se surestiment, $50 \%$ s'évaluent correctement et 10/20\% se sous-estiment (Mynttinen et al., 2009 a et b) ; donc, nous voyons que tous les jeunes conducteurs novices ne nécessitent pas une amélioration de l'autoévaluation et une diminution de la surconfiance !

Comme évoqué plus haut, l'entraînement avec des formateurs professionnels semble réduire la surconfiance ; celle-ci diminue d'ailleurs avec l'expérience de conduite : ce sont les conducteurs très inexpérimentés qui se surestiment fortement, puis, avec l'expérience, les autoévaluations suivent les progrès des compétences (Boccara et al., 2011 a et b).

Des programmes d'amélioration de l'autoévaluation (ou d'insight) pourraient donc diminuer la surconfiance, des évaluations ont apporté des résultats prometteurs (Gregersen, op. cit.) mais des carences méthodologiques importantes subsistent.

\section{Conclusion}

La mise en évidence d'un impact d'une formation sur le risque accidentel ultérieur des jeunes conducteurs novices n'apparaît pas comme évidente du fait de multiples difficultés d'ordre méthodologique (manque fréquent de groupe témoin, de contrôle des biais de sélection des échantillons, de suivi suffisamment long et précis sur les accidents et les infractions, etc.) ; par contre, des améliorations du comportement de conduite ont pu être observées. Certains types de formation (par exemple, les formations au pilotage) améliorent les compétences sans diminuer le risque, d'autres types de formation (par exemple, les entraînements aux "compétences de vie ») améliorent le risque sans influencer les compétences. 

approches pédagogiques en les répartissant entre avant et après le permis, mais en fait, intégrer les quatrième et cinquième niveaux de la matrice dans la formation à la conduite supposera de faire descendre en formation initiale des méthodes qui sont utilisées en post-permis (comme par exemple les jeux de rôle, les discussions de groupe, les questionnaires d'autoévaluation, etc.) pour améliorer l'auto-évaluation des conducteurs novices à propos de leurs problèmes de vitesse, d'alcool, de cannabis, de téléphone, de stress, etc. Nos futurs travaux évaluant les actions de sensibilisation menées actuellement dans les lycées devraient par exemple apporter des éléments de réflexion, et de nombreuses autres recherches pourraient être menées dans ce domaine, notamment sur l'appariement entre type de conducteur et type de programme éducatif ou préventif. 


\section{BIBLIOGRAPHIE}

Allen, R.W., Park, G.D. (2007). The effect of driving simulator fidelity on training effectiveness. In: Proceedings of the Driving Simulator Conference North America, Iowa City.

Assailly, J.P. (2001). La mortalité des jeunes, Que sais-je ?, PUF : Paris.

Assailly, J.P. (2007). Jeunes en danger. Les familles face aux conduites à risques, Imago : Paris.

Assailly, J.P. (2010). La psychologie du risque. Lavoisier, Collection Sciences du Risque et de la

Décision : Paris.

Boccara, V., \& Delhomme, P. (2011a). Development of student drivers' self-assessment accuracy during French driver training: self assessments compared to instructors' assessments in three risky driving situations, Accident Analysis \& Prevention, 43, 1488-1496.

Boccara, V., \& Delhomme, P. (2011b). Time course of driving-skill self-assessments during French driver training, Accident Analysis \& Prevention, 43, 241-246.

Boufous, S., \& Ivers, R. (2011). Attempts at the practical on-road driving test and the hazard perception test and the risk of traffic crashes in young drivers. Traffic Injury Prevention, 12, 475482.

Carstensen, G. (2002). The effect on accident risk of a change in driver education in Denmark, Accident Analysis \& Prevention, 34, 111-121.

Crundall, D., \& Andrews, B. (2010). Commentary training improves responsiveness to hazards in a driving simulator, Accident Analysis \& Prevention, 42, 2117-2124.

De Winter, J.C.F., \& Wieringa, P.A. (2007). Violations and errors during simulator-based driver training. Ergonomics, 50, 138-158.

De Winter, J.C.F., \& de Groot, S. (2009). Relationships between driving simulator performance and driving test results. Ergonomics, 52, 137-153.

Fisher, D.L., \& Pollatsek, A.P. (2006). Can novice drivers be trained to scan for information that will reduce their likelihood of a crash? Injury Prevention, 12, i25-i29.

Gregersen, N.P. (1996). Young drivers' overestimation of their own skill-an experiment on the relation between training strategy and skill, Accident Analysis \& Prevention, 28, 243-250.

Hatakka, M., Keskinen, E., Gregersen, N.P., Glad, A., \& Hernetkoski, K. (2002). From control of the vehicle to personal self-control: broadening the perspectives to driver education, Transportation Research Part F, 2002, 201-215.

Hirsch, P., Maag, U., \& Laberge-Nadeau, C. (2006). The role of driver education in the licensing process in Quebec, Traffic Injury Prevention, 7, 130-142.

Isler, R.B., \& Starkey, N.J. (2011). Effects of higher-order driving skill training on young, inexperienced drivers' on-road driving performance, Accident Analysis \& Prevention, 43, 1818-1827.

Katila, A., \& Keskinen, E. (1996). Conflicting goals of skid training, Accident Analysis \& Prevention, 28 , 785-789. 
Katila, A., \& Keskinen, E. (2004). Does increased confidence among novice drivers imply a decrease in safety? The effects of skid training on slippery road accidents, Accident Analysis \& Prevention, 36, 543-550.

Kelly, M.J. (2005). Effects of Defensive Vehicle Handling Training on Novice Driver Safety: Phase 1. Preparation for Advanced Driver Training. Western Transportation Institute, Bozeman, MT.

King, K.A., \& Vidourek, R.A. (2008). Teaching adolescents safe driving and passenger behaviors : effectiveness of the You Hold the Key Teen Driving Countermeasure, Journal of Safety Research, 39, 19-24.

Lund, A.K., Williams, A.F., \& Zador, P. (1986). High school driver education: further evaluation of the DeKalb County study, Accident Analysis \& Prevention, 18, 349-357.

Mayhew, D.R., Simpson, H.M., \& Pak, A. (2003). Changes in collision rates among novice drivers during the first months of driving, Accident Analysis \& Prevention, 35, 683-691.

Mynttinen, S., \& Sundström, A. (2009a). Are novice drivers overconfident? A comparison of selfassessed and examiner-assessed driver competences in a Finnish and Swedish sample, Transportation Research Part F, Traffic Psychology Behavior, 12, 120-130.

Mynttinen, S., \& Sundström, A. (2009b). Self-assessed driver competence among novice drivers-a comparison of driving test candidate assessments and examiner assessments in a Dutch and Finnish sample, Journal of Safety Research, 40, 301-309.

Nyberg, A., Gregersen, N.P., \& Wiklund, M. (2007). Practicing in relation to the outcome of the driving test, Accident Analysis \& Prevention, 39, 159-168.

ONISR (2011). Les accidents corporels de la circulation. Document de travail. www.sécuritéroutiere.fr Petersen, A., \& Barrett, R. (2006). Driver-training and emergency brake performance in cars with antilock braking systems, Safety Science, 44, 905-917.

Russell, K.F., Vandermeer, B., \& Hartling, L. (2011). Graduated driver licensing for reducing motor vehicle crashes among young drivers. Cochrane Database Syst. Rev. (10) (Art. No. : CD003300).

Senserrick, T., \& Ivers, R. (2009). Young driver education programs that build resilience have potential to reduce road crashes, Pediatrics, 124, 1287-1292.

Tronsmoen, T. (2010). Associations between driver training, determinants of risky driving behaviour and crash involvement. Safety Science, 48, 35-45.

Underwood, G., \& Crundall, D. (2002). Selective searching while driving : the role of experience in hazard detection and general surveillance. Ergonomics, 45, 1-12.

Wang, Y., \& Zhang, W. (2010). Effects of a simulation-based training intervention on novice drivers' hazard handling performance. Traffic Injury Prevention, 11, 16-24.

\section{RÉSUMÉS}

Cet article présente une revue des diverses innovations pédagogiques apparues dans les pays industrialisés afin de réduire le surrisque des jeunes conducteurs novices, ainsi que de leurs évaluations. On présente d'abord les logiques scientifiques et politiques qui ont conduit certains pays à se focaliser sur la diminution de l'exposition au risque (accès graduel à la conduite ou permis progressif) ou d'autres sur l'éducation du conducteur afin de renforcer ses diverses compétences lors de cette phase d'inexpérience. À partir du champ théorique et méthodologique impulsé à partir de la matrice GDE, on passe en revue les divers types de formation, avant le 
permis (apprentissage avec les enseignants de la conduite, conduite accompagnée, entraînement sur simulateur) et après (entraînements aux manœuvres de pilotage, formations cognitives, entraînement à la perception des dangers, conduite commentée, complètement de tâche, entraînements des " compétences de vie ", programmes d'amélioration de l'autoévaluation). On conclura par les enseignements de cette revue à propos des bonnes pratiques visant à modérer la confiance excessive, en développant les compétences méta-cognitives (autoévaluation plus réaliste de soi) à chaque niveau de la matrice GDE

This paper presents a review of the various innovations in driver training methods which have been elaborated in the industrialized countries, in order to reduce the overrisk of young novice drivers, and of their evaluations. First, we present the scientific and policy factors that have guided some countries to focus on the reduction of risk exposure (GDL or graduated driver licensing) and other countries on driver education, in order to improve the various driving and safety skills during this phase of inexperience. Starting from the theoretical and methodological field of the GDE matrix, the various types of training are reviewed, before licensing (training with professional driving teachers, accompanied driving, simulator training) and after licensing (practical driving skills training, cognitive training like risk perception training, commented driving, task completion, life-skills training, self-evaluation training). Last, we conclude on what we have learnt about good practices concerning moderation of excessive confidence and development of meta-cognitive skills (more realistic self-evaluation) at each level of the GDE matrix.

\section{INDEX}

Mots-clés : formation, risque, jeunes conducteurs, méthodes pédagogiques, compétences de vie Keywords : driver training, risk, young drivers, training methods, life-skills

\section{AUTEUR}

\section{ASSAILLY JEAN-PASCAL}

IFSTTAR, LPC 